\section{A Quick, Economical and Gentle Method for Applying Small Volumes of Solutions to Specimens on Glass Slides}

Clare Hasenkampf

University of Toronto at Scarborough

I use immunocytochemical detection schemes that employ the use of primary and secondary antibodies for either tissue sections or whole mount preparations in order to study the in situ distribution of proteins and labeled nucleic acids. As the antibodies are either laboriously generated, or represent costly purchases, I have sought a protocol that minimizes the volume of antibody solution needed. Additionally I need a protocol that involves a quick, thorough, and gentle application and removal of the antibody solutions from the specimen with no danger of the specimen drying out during the one or two hour incubations. After trying many various schemes I have settled into the use of a procedure which very effectively retains the antibody solution on the specimen without damaging the delicate sections or whole mounts, and which is easy to add and remove.

My starting material typically is paraffin embedding $5-10 \mu \mathrm{m}$ sections of lily anthers. I use three sections per slide, and the sections are placed slightly off center. Alternatively I also use whole mount chromosome preparations which are located in the center of a microscope slide within a diameter of $1.5 \mathrm{~cm}$.

I perform the blocking step and antibody reactions by placing the sections (or whole mounts) upside down on top of the solution on a hydrophobic surface. I keep the sections from actually touching the hydrophobic surface by having them suspended across a platform made of two other microscope slides. I have found this technique to be a very reliable, quick and inexpensive way to process my slides gently for immunocytochemistry. I do the antibody incubations for 2 hours and have not had any problems with the solutions drying up or spreading out too far.

The method of setting up the hydrophobic platform area is as follows.

Prepare a hydrophobic work area as follows:

- Pull off a large piece of heavy duty plastic wrap (for example microwavable HandiWrap). Be careful to touch only the edges of the wrap.

- Starting at one end, begin to tape the plastic wrap to a level lab bench surface.

- Proceed to the opposite end, pulling the wrap very taut as you go. Remember touch only the edges of the plastic wrap.

When you have finished you should have a taut, smooth surface with no wrinkles.

\section{Once you have the hydrophobic surface,} it's time to create the platforms:

For this you'll need ordinary clean glass microscope slides.

- Take the first microscope slide and place it in the top left corner of your plastic wrap work surface, leaving at least a 1.5 inch border from both top and side edge.

- Now place another microscope slide parallel to it with a distance between the two slides of approximately 1.5 inch.

To apply the solution to the experimental slides:

- Using a micropipette place the desired solution (I use 100-200 $\mu \mathrm{ls}$ ) on the plastic wrap in the upper, middle region between the two slides of the platform.

- Once the liquid is in place, carefully lower the slide with sections onto the drop, tissue side down.

This should place the sections in contact with the liquid and spread the liquid out somewhat, but the sections will not actually contact the surface of the plastic wrap.

Each such set of two platform slides can be used as the platform to process two slides for immunocytochemistry for one treatment, Figure 1. Thus if you have 10 slides in your experiment you'll need 5 platforms per solution you wish to apply.
To remove the solution from the experimental slide:

- Holding the slide of the relevant portion of the platform, simply lift the experimental slide from the drop, shake it once briskly (there is very little liquid on the slide) and quickly move the slide to its next solution.

I do both my blocking step and my antibody incubations on these platforms. I use $200 \mu$ ls of blocking reagent at the blocking step, and $100 \mu \mathrm{ls}$ of the antibody mixtures. In this way the region of the slide covered with blocking solution is larger than the region covered by the antibody solution and this helps ensure proper blocking of the slide.

\section{Avoiding problems}

1. The spacing of the two platform slides is important. The specific distance to place them apart will depend on where the sections are on the slide, how much of the slide's area the sections occupy, and how much solution you want to use. You can use trial and error with a mock hybridization solution to optimize the spacing for each type of sample.

2. Do not use the same region of the plastic wrap twice. Use a fresh region for each new incubation.

3. Try to get the plastic wrap exactly like you want it before you add any liquid. Trying to pull the wrap tighter after the liquid as been applied can cause dramatic changes in the liquid's location.

4. Before placing the sections on the slide platform, most of any previous solution must have been removed. If the slide is too wet, the antibody solution may spread out too much as it merges with residual liquid.

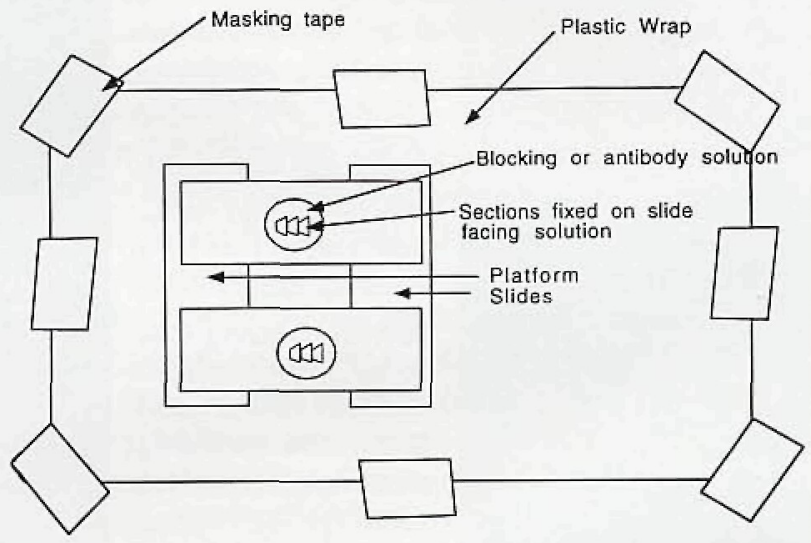

Figure 1: Platform above hydrophobic surface for small volume incubations.

\section{PROTOCOL OF PLANT SPECIMEN PREPARATION FOR TRANSMISSION ELECTRON MICROSCOPY Continued from Page 18}

10. Infiltrate in stoppered vial(s) with equal parts of propylene oxide and resin mixture 6-8 hours (or overnight) on rotator. Store the samples in a dry location, but not in a completely sealed chamber where propylene oxide fumes may accumulate dangerously.

11. Infiltrate in unstoppered vial(s) with one part propylene oxide, and three parts resin mixture. Continue running vial(s) on rotator for $8-12$ hours. Store the samples in a dry location, but not in a completely sealed chamber where propylene oxide fumes may accumulate dangerously.

12. Transfer to pure resin mixture for $1-4$ hours while rotating.

13. Embed specimens in BEEM ${ }^{T M}$ capsules or in flat embedding molds. BEEM also makes a hard flat mold that fits into a plastic case. Remove the case before putting specimens in the oven. These molds are excellent and can be used over a number of times. If using BEEM capsules, close the lids. If using flat molds, place each one inside of a covered petri dish. Place in $70^{\circ} \mathrm{C}$ oven for 36 hours. At the end of that time, remove the specimens and allow them to cool for $c a$. 15 minutes prior to checking for correct hardness with razor blade. If too soft, return to the oven for an additional 12-24 hours.

14. Trim and section with the sharpest tools available. Avoid stain precipitates in post-staining techniques. Usually, 10 minutes of $5 \%$ aqueous (or methanolic) uranyl acetate, followed by washing and 5-10 minutes of Reynold's lead citrate staining is sufficient. 

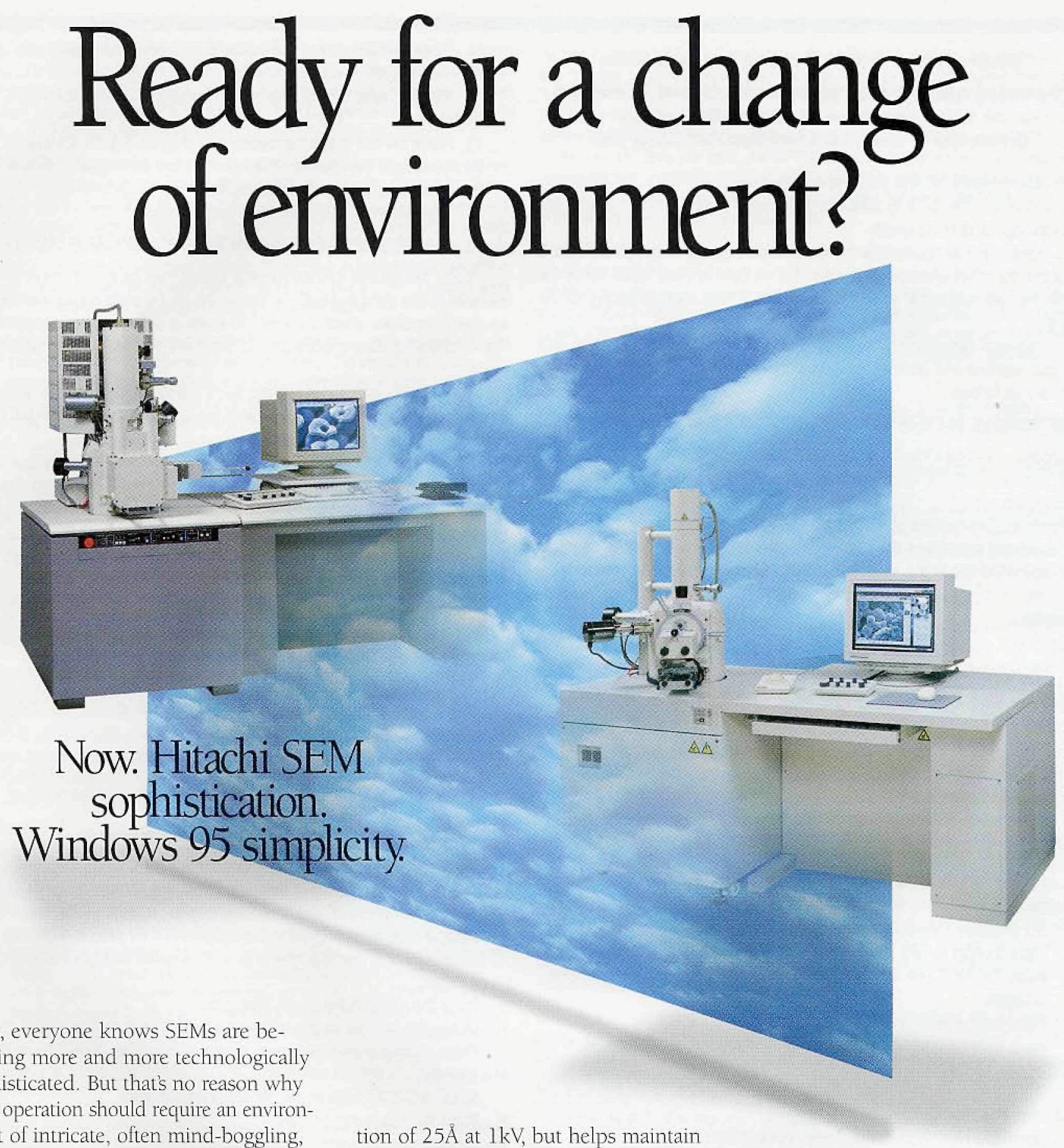

Sure, everyone knows SEMs are becoming more and more technologically sophisticated. But that's no reason why their operation should require an environment of intricate, often mind-boggling, challenges.

Enter Hitachi's new S-4700 and S-3500N SEMs, which replace all that operational complexity with the easy familiarity of Windows ${ }^{\mathrm{TM}} 95$ control. So anyone who's ever used a Windowsbased PC can - with a near-zero learning curve - take over their operation. And how's this? To keep die-hard traditionalists just as excited, these SEMs can come set up for convenient switching to conventional control as well.

Our S-4700 FE SEM and S-3500N Variable Pressure SEM offer even more to be excited about. Like superior resolution. The 5-4700's new objective lens, for example, not only contributes to a resolu- tion of $25 \AA$ at $1 \mathrm{kV}$, but helps maintain that high resolution even when studying large samples at high tilt angles and at long working distances. Further, both instruments are available with our $\mathrm{Hi}$ Mouse feature, for operating EDX systems right from the SEM. (In fact, the S-4700's analytical mode permits EDX operation concurrent with high-resolution imaging.) And, with our Rapid Image Shift Movement (RISM) capability, you'll have the fastest, most precise specimen navigation ever.

So. Super performance made super easy. It's surely a refreshing environment for SEM microscopy. And just as surely, you'll want details on these breathrough SEMs. You can get them easily by phone or E-mail, or by visiting our Web sitewhich, of course, you'll very likely do via a friendly Windows environment.

\section{HITACHI}

SCIENTIFIC INSTRUMENTS

Nissei Sangyo America, Ltd.

755 Ravendale Drive

Mountain View, CA 94043

(800) $227-8877$

E-mail: sidsales@nissei.com www.nissei.com

25 West Watkins Mill Road Gaithersburg, MD 20878 (800) 638-4087

Windows is a registered tridemark of Microsoft Corporation 